\title{
Bdellovibrio Like Organisms: The Predatory Assassin
}

\author{
Aditya Sinha ${ }^{1}$, Megha Hurakadli ${ }^{2}$, Shivamurthy Ravindra ${ }^{3}$, Amita Agarwal ${ }^{4}$ \\ ${ }^{I}$ Department Of Periodontics, K.D.Dental College \& Hospital,India \\ ${ }^{2}$ Department Of Oral And Maxillofacial Surgery, Medlife Foundation,India \\ ${ }^{3}$ Department Of Periodontics, Sri Hasanamba Dental College \& Hospital,India \\ ${ }^{4}$ Department Of Periodontics, K.D.Dental College \& Hospital,India
}

\begin{abstract}
Periodontitis is a multifactorial and a polymicrobial infectious disease primarily associated with gram negative bacterias. Bdellovibrio and like organism (BALOs) are small, fast, flagellate, deltaproteobacteria that prey on other gram negative pathogens. It utilizes the cell content, including DNA and $R N A$ for its growth. This significant activity has been the focus of research for many years, with useful practical applications in both dental and medical fields. Bdellovibrio has potential as a bio control agents or living antibiotics. This review article focuses on the life cycle of bdellovibrio and its use against the elimination of harmful and pathogenic bacteria, highlighting its future role and implication in periodontal diseases.
\end{abstract}

Keywords: Bdellovibrio, Periodontal disease, Periodontitis, Predator, Prey

\section{Introduction}

In the ecosystem, predation is a unique interaction where a prey (the organism that is being attacked) is being fed upon by a predator (an organism that is hunting). Predation is prevalent in all fields of life and possibly in all realms of environments. In natural environments bacteria are subjected to predation from bacteriophage, protists and predatory prokaryotes. Predatory prokaryotes are distinctive in the fact that they are bacterium, a living organism, as opposed to viruses and phages, and are smaller than the prey, in contrast with protists.

Planktonic free swimming bacteria are different from biofilm bacteria as they have ability to form microbial communities with distinct biochemical and phenotypic properties [1]. Bacteria sense favorable environmental conditions that activate the transition to life on a surface, followed by a multiple sequential step which leads to colonization of bacteria to form a mature biofilm [2,3]. Periodontitis is a polymicrobial infection involving numerous gram negative pathogens embedded in a complex biofilm called dental plaque. It is multifactorial infection caused by a complex of bacterial species predominantly including gram negative bacteria that interact with host tissues and causes destruction of the periodontal structures and the toothsupporting tissues. The importance of bacteria in dental plaque and the key role of plaque in the causation of periodontal disease is well established. Whereas oral bacteria colonize and produce disease primarily in the oral cavity, they can also produce systemic disease. Therefore, the mitigation of oral infection is of broad clinical importance beyond the boundaries of the oral cavity. The difficulty in removing oral plaque or biofilms by conventional therapies led researchers to examine other alternative methods for biofilm prevention, such as biological inhibitory or control agents. One such agent that might be used to control pathogenic bacteria is the predatory prokaryotes from the genus Bdellovibrio. Bdellovibrio are gram-negative bacteria that feed on other gram-negative bacteria. They were discovered by chance in 1962 by Stolp and Petzold while they were trying to isolate bacteriophage for plant pathogenic bacteria from soil [4]. There are three species of BdellovibrioB.bacteriovorus, B. stolpii and B.Starrii.

\section{Morphology Of Bdellovibrio Bacteriovorus}

Bdellovibrio bacteriovorus is a tiny obligate predator of gram negative bacteria $(0.2-0.5 \mu \mathrm{m} \times 0.5-2.5$ $\mu \mathrm{m})$ universally found in bulk soil, in the rhizosphere of plants, and in freshwater bodies such as rivers and water treatment facilities [5]. Due to their ability to prey upon bacteria, including many pathogens of humans and plants, they have been termed "living antibiotics" [6]. Bdellovibrio bacteria are fast and flagellated bacteria, swimming at high speeds (60 to $160 \mu \mathrm{m} / \mathrm{s}$ ) by rotating a single polar flagellum, using chemotaxis to locate regions rich in prey bacteria [7]. 


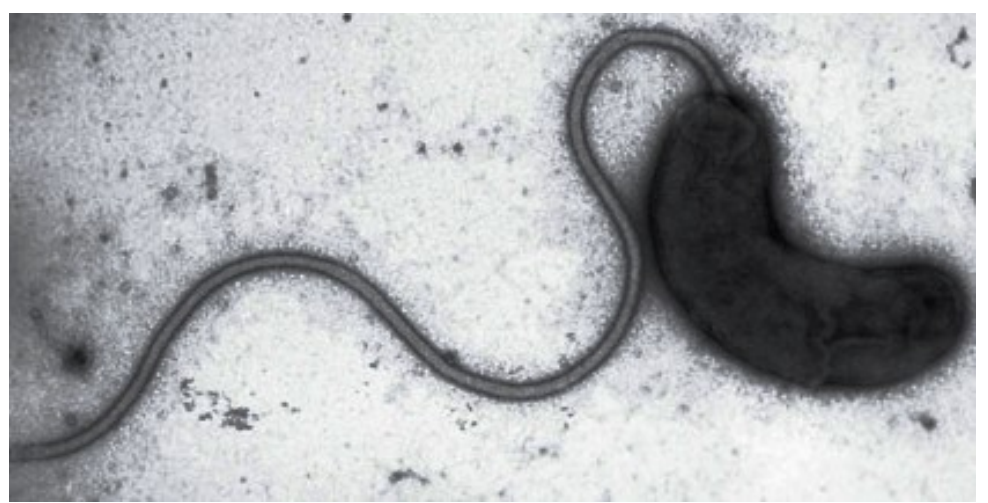

Fig.1 Bdellovibrio Bacteriovorus

B.bacteriovorus are deltaproteobacterium that displays a dimorphic life cycle, with two main phenotypes: Attack Phase (AP) and Growth Phase (GP). AP cells are small, fast swimming, non-replicating "hungry" cells that search for prey, GP cells are non-motile replicative cells that actively consume the prey [8]. To enter the prey cell periplasm, a bdellovibrio must penetrate both the prey cell outer membrane and the covalently linked peptidoglycan. Although mechanical action at the point of attachment may contribute to penetration, especially through the outer membrane, a variety of enzymatic reactions accompany penetration $[9,10]$ and apparently play a significant role in the process. Included in these enzymatic activities are the activity of a lipopolysaccharidase and activities of three enzymes directed against the prey peptidoglycan: a glycanase which rapidly solubilizes about $10 \%$ of the $\mathrm{N}$-acetylglucosamine during penetration; a deacetylase which deacetylates the peptidoglycan, rendering it insensitive to further attack by the glycanase; and a peptidase that releases soluble diaminopimelic acid(DAP) residues throughout the intraperiplasmic life cycle of the bdellovibrio $[9,10]$.

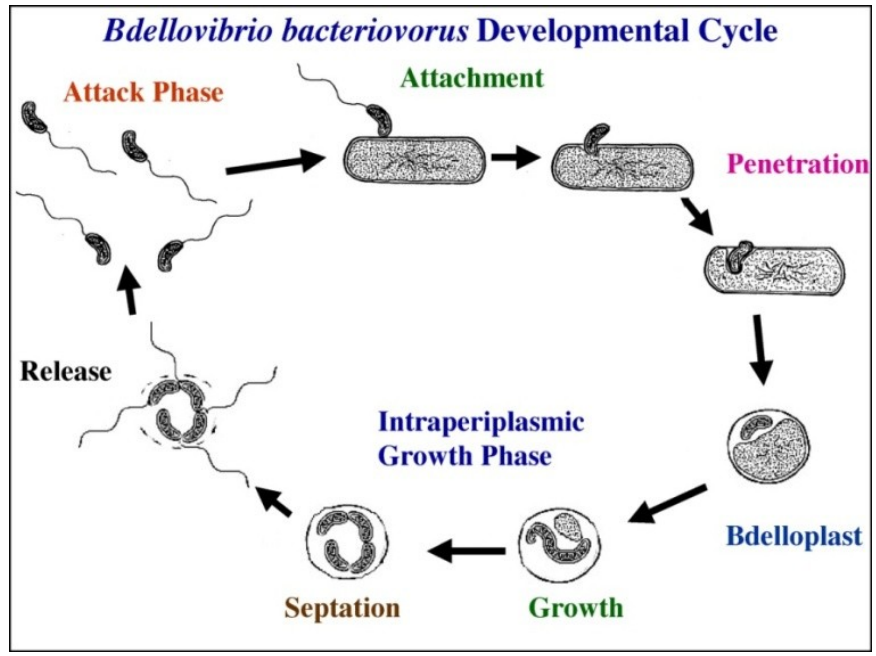

Fig.2 Life cycle of bdellovibrio bacteriovorus

B. bacteriovorus obtains its energy and nutrients from the cytoplasmic content of its prey cells, it grows and replicates within the periplasm of the prey cells. Prior to growth, the prey cell is killed and remodelled into a spherical bdelloplast. Growth within the prey cell takes place as an aseptate and polynucleoid filament with cell division controlled by the availability of soluble host components [11]. Three hours after the initial attachment to the prey, bdelloplast exhaustion and division of elongated B. bacteriovorus takes place, the GP cycle of B. bacteriovorus lasts about $4 \mathrm{hrs}$, with the prey being killed within 30 minutes [12]. The cycle is completed by a subsequent rupturing of the bdelloplast envelope and a release of daughter AP cells.

\section{Advantages Offered By BALOs}

\subsection{Effect Of Bdellovibrio And Like Organisms (BALOs) On Pathogenic Bacteria}

BALOs show a very wide range and non-specific predation of their host cells. They can attack gram negative bacteria from very diverse and different genera. It was reported that the Bdellovibrio bacteriovorus 109J host range includes strains from Escherichia, Pseudomons, Chromatium and Spirillum including Acinetobacter, Aeromonas, Bordetella, Burkholderia, Citrobacter, Enterobacter, Klebsiella, Listonella, 
Morganella, Proteus, Serratia, Salmonella, Shigella, Vibrio and Yersinia [13]. Proving that many pathogenic gram-negative bacterial strains which infect humans, in addition to animal and plant pathogens, can be predated upon by one or more strains of Bdellovibrio.

Bdellovibrios are highly specific for infecting bacteria and thus are harmless to nonbacterial organisms. As the bdellovibrios can multiply in the host cell releasing new progeny upon lysis, the intial dose of bdellovibrio can be kept low.

\subsection{Effect Of Bdellovibrio And Like Organisms (BALOs) On Biofilms}

Most of the bacterial chronic inflammatory and infectious diseases in humans involve biofilms formation, made by uropathogenic E. coli in urinary tract infections, enterohemorragic E. coli in gastrointestinal infections, and wound, burn and respiratory infections caused by P. aeruginosa even periodontitis which is a polymicrobial infection involving numerous gram negative pathogens embedded in a complex biofilm called dental plaque. In general, the main complication resulting from biofilm formation is that bacteria inside these biofilms are protected well and become much more resistant to antibiotic treatment as compared to planktonic cells $[14,15]$ and this, in turn, contributes to the further progression of the infection. Bdellovibrio with their ability to invade biofilms, penetrating them deeply and effectively destroying them, as demonstrated in the studies done by Kadouri et al. It was found that Bdellovibrio was even more efficient in attacking biofilms than the planktonic cells, leading to a greater loss in bacterial counts, supposedly due to the higher prey density in biofilms and hence faster and easier access to the prey. B. bacteriovorus was also able to attack biofilms made by single species and mixed species and does have the capability to access biofilms as thick as $30 \mu \mathrm{m}$ and is not restricted to the surface of the biofilm $[16,17]$

\subsection{Effect Of Bdellovibrio And Like Organisms (BALOs) On Periodontal Health}

In recent studies done by Van Essche et al [18], Bdellovibrio bacteriovorus HD100 could attack Aggregatibacter actinomycetemcomitans (one of the major species which contribute to periodontitis). In a successive study by the same group [19], they showed that different bacterial species involved in periodontitis can be attacked by one or more of Bdellovibrio strains, even if the prey was strict anaerobe. Scanning electron microscope revealed Bdellovibrio was capable of decreasing the biofilm biomass.

Dashiff and Kadouri demonstrated that B. bacteriovorus predation of biofilms made by oral pathogens ,i.e., Eikenella corrodens and A. actinomycetemcomitans, they found that B. bacteriovorus could eliminate biofilms developed on hydroxyapatite surfaces and it could also attack metabolically inactive biofilms. Another important finding in this study was its ability to attack in presence of saliva [20]. This will be of great significance for the use of BALO in the future as an oral antibacterial agent in mouthwashes, gargles, etc. But no predation was observed against P.gingivalis, P.intermedia, T.forsythia and F.nucleatum ATCC10953.

Van Essche et al.[19] evaluated the predation efficiency of six different BALO strains (B.bacteriovorusHD100, B. bacteriovorus109J, BacteriovorusBEP2, Bacteriovorax FCE, PeredibacterstarriiA3.12, BacteriovoraxstolpiiUki2) against six different perioodontopathogens (A. actinomycetemcomitans, E.corrodens, P. gingivalis, C.sputigena, P. intermedia, F. nucleatum). Among the six different strains of BALOs, B.bacteriovorusHD100 had the widest prey spectrum by decreasing the viability of four tested periodontopathogens.The other strains like B. bacteriovorus 109J caused lysis of three perioodontopathogens, Bacteriovorus BEP2 and Bacteriovorax FCE was effective two tested pathogens. P.starrii A3.12 was effective against A.actinomycetemcomitans. B.stolpii Uki2 did not predate any of the tested preybacteria. This highlights the concept that the predator - Prey interactions are highly BALO strain specific and oral application of BALO strains at high inoculum concentrations has the potential to rapidly decrease the numbers of a wide range of periodontal pathogens from the mixed oral microbiota.

\subsection{Effect Of Bdellovibrio And Like Organisms (BALOs) On Multidrug Resistant Gram Negative Pathogen}

Pathogenic multidrug-resistant (MDR) bacteria have emerged as a serious hazard to the well being of humans. Particularly concerned are MDR Gram-negative bacteria producing highly potent $\beta$-lactamases such as the extended-spectrum ß-lactamase and KPC-type B-lactamase [21]. An alternative approach to combat antimicrobial-resistant bacterial infections is the use of predatory bacteria to eliminate MDR pathogens. Kadouri et al evaluated the predatory action of B.bacteriovorus 109J, B.bacteriovorus HD100 and M.aeruginosavorus ARL-13 against thirteen MDR gram negative pathogen \{Acinetobacter baumannii (AB276,AB285), Escherichia coli

(YD429,YD438,YD446,YDC345,AZ1285)Klebsiellapneumonia(YD466,AZ1032,AZ1093,AZ1136,A Z1169), pseudomonas aeruginosa GB 771, Pseudomonas putida YA241 ).It was demonstrated that bacteriovorus HD100 was able to prey on all examined host bacteria, B. bacteriovorus 109J was able to prey on 13 of the 14 host bacteria Five out of the 7 examined host bacteria were reduced by M. aeruginosavorus ARL-13.The 
predators maintained their ability to prey on the host cells despite the MDR status and highlighted the potential application of predatory bacteria as a biological control agents[21].

$\beta$-lactam antibiotics have no effect on BALO thus, they can be used in conjunction with these antibiotics since the predatory strains will still be active and attack the prey species. This characteristic is clearly beneficial with an antibiotic resistant strain.

BALO has specific type of resistance which is due to a plastic phenotype rather than permanently genetic encoded and till date, no completely resistant mutant prey has been found against the predatory bacteria. The fact that the resistance to BALOs is lost quickly proposes that they utilize a cellular component necessary for survival of the prey and thus their predation

\section{Demerits Of BALOs}

- Gram negative bacteria with S-layer on their surfaces are resistant to BALOs attack. Their ability to attack is affected by the physiological status of their prey and there activity is also interfered and decreased or even increased by the presence of other bacteria [22].

- BALOs fail to completely kill their prey, even at high predator to prey ratio.

- As these predatory bacterias are strict aerobes limiting their potential use in oxygen reduced/anaerobic environments, such as gut, urinary tract or periodontal pockets

- High concentrations of glucose or glycerol and also low pH affect BALOs activity. These positive and negative interactions with the surrounding environment affect the efficiency of predation in real application.

- At first glance it would seem to be a beneficial trait, such as when mixed bacterial species are present in the infections, as happens in cystic fibrosis [23]. However, since their effects on the natural flora of different body cavities has not been studied thoroughly and should be taken into consideration, the use of BALOs in this case would need to be monitored.

- BALOs are unable to attack or predate upon Gram-positive strains, a class that consist of many human pathogens including Staphylococcus aureus, which is one of the most common nosocomial infectionassociated multidrug resistant pathogens isolated from patients [24].

\section{Future Use Of BALOs In Treatment Of Periodontal Disesase}

- Bdellovibrio has the ability to access biofilm hence can be used to inhibit plaque formation, which is primary etiological agent for chronic periodontitis.

- Chronic periodontitis involves various gram negative microflora, they can be combated using Bdellovibrio.

- It can be used for treatment of Aggressive periodontitis which are caused by gram negative bacteria, A.actinomycetemcomitans and P.gingivalis.

- Bdellovibrio are unaffected by $\beta$-lactam antibiotics, they can be used as an adjunct to local drug delivery and systemic antibiotics for nonsurgical treatment of periodontal pocket, gingival and periodontal abscess.

- Can be used to kill multi drug resistance gram negative bacteria that tend to develop due repeated use of antibiotics.

\section{Conclusion}

Multi drug resistant pathogen are becoming a major clinical concern as they can no longer be treated adequately by conventional antimicrobial agents, the concept of using predatory bacteria as live anti microbial is gaining popularity. Even oral application of BALO strains at high inoculum concentrations has the potential to rapidly decrease the numbers of a wide range of periodontal pathogens from the mixed oral microbiota. Hence predatory therapy can therefore be regarded as an interesting opportunity for the development of an adjuvant to standard periodontal therapy. These minute assassins offer a promising path for the treatment of diseases related to biofilms and in particular, periodontal infections. Its widespread applications in future provides opportunity for further research in both medical and non medical field.

\section{References}

[1]. Costerton, J. W.et al, Microbial biofilms, Annual Review of Microbiology, 49, 1995,711-745.

[2]. Watnick, P. and Kolter, R, Biofilm, city of microbes, Journal of Bacteriology, 182, 2000, $2675-2679$.

[3]. Palmer, R. J and White, D.C, Developmental biology of biofilms: implications for treatment and control, Trends in Microbiology, $5,1997,435-440$.

[4]. Stolp, H. and Starr, M. P, Bdellovibrio bacteriovorus Gen. Et Sp. N., a Predatory, Ectoparasitic, and Bacteriolytic Microorganism, Antonie Leeuwenhoek 29, 1963, 217-248.

[5]. Jurkevitch, E.et al, Prey range characterization, ribotyping, and diversity of soil and rhizosphere Bdellovibrio spp. isolated on phytopathogenic bacteria, Applied and Environmental Microbiology, 66, 2000, 2365-2371.

[6]. Sockett, RE. and Lambert C, Bdellovibrio as therapeutic agents: a predatory renaissance?, Nature Reviews Microbiology, 2, 2000, $669-675$.

[7]. Iida, Y.et al, Roles of multiple flagellins in flagellar formation and flagellar growth post bdelloplast lysis in Bdellovibrio bacteriovorus, Journal of Molecular Biology 394, 2009, 1011-1021. 
[8]. Sockett, RE, Predatory lifestyle of Bdellovibrio bacteriovorus, Annual Review of Microbiology, 63, $2009,523-539$.

[9]. Thomashow, M.F. and Rittenberg, S.C, Intraperiplasmic growth of Bdellovibrio bacteriovorus 109J: solubilization of Escherichia coli peptidoglycan, Journal of Bacteriology, 135, 1978, 998-1007.

[10]. Thomashow, M. F. and Rittenberg, S.C, Intraperiplasmic growth of Bdellovibrio bacteriovorus 109J: N-deacetylation of Escherichia coli peptidoglycan amino sugars, Journal of Bacteriology, 135, 1978, 1008-1014.

[11]. Kessel, M. and Shilo, M, Relationship of Bdellovibrio elongation and fission to host cell size, Journal of Bacteriology, 128, 1976, 477-480.

[12]. Lambert, C. et al, Bdellovibrio: growth and development during the predatory cycle, Current Opinion in Microbiology 9, 2006, 639-644.

[13]. Dashiff, A. et al, Predation of human pathogens by the predatory bacteria Micavibrio aeruginosavorus and Bdellovibrio bacteriovorus, Journal of Applied Microbiology, 110, 2011, 431-444.

[14]. Stewart, P. S. and Costerton, J. W, Antibiotic resistance of bacteria in biofilms, Lancet, 358, 2001, $135-138$.

[15]. Stewart, P. S, Mechanisms of antibiotic resistance in bacterial biofilms, International Journal of Medical Microbiology 292, 2002, 107-113,

[16]. Kadouri, D. and O'Toole, G. A, Susceptibility of biofilms to Bdellovibrio bacteriovorus attack, Applied Environmental Microbiology, 71, 2005, 4044-4051.

[17]. Dashiff, A. et al, Predation of human pathogens by the predatory bacteria Micavibrio aeruginosavorus and Bdellovibrio bacteriovorus, Journal of Applied Microbiology, 110, 2011, 431-444.

[18]. Van Essche, M. et al, Bdellovibrio bacteriovorus Attacks Aggregatibacter actinomycetemcomitans, Journal of Dental Research, 88, 2009, 182-186.

[19]. Van Essche, M. et al, Killing of anaerobic pathogens by predatory bacteria, Molecular Oral Microbiology 26, $2011,52-61$.

[20]. Dashiff, A. and Kadouri, D. E, Predation of oral pathogens by Bdellovibrio bacteriovorus 109J. Molecular Oral Microbiology,26, 2011, 19-34.

[21]. Kadouri D.,To K.,Shanks R.,Doi Y. Predatory bacteria: A potential ally against multidrug-resistance gram negative pathogens, Plos ONE, 8(5):e63397.

[22]. Dwidar, M. et al, The dual probiotic and antibiotic nature of Bdellovibrio bacteriovorus. Biochemistry and Molecular Biology Reports, 45, 2012,71-78.

[23]. Govan, J. R. W. and Deretic, V, Microbial pathogenesis in cystic fibrosis: Mucoid Pseudomonas aeruginosa and Burkholderia cepacia, Microbiology Review, 60, 1996, 539-574.

[24]. Valaperta, R. et al, Staphylococcus aureus nosocomial infections: the role of a rapid and low-cost characterization for the establishment of a surveillance system, New Microbiologica, 33,2010, 223-232. 